\title{
Evaluation of a new bi-valve vaginal speculum applicator design for gynecologic interstitial brachytherapy
}

\author{
Hiroaki Kunogi, MD, PhD, Nanae Yamaguchi, MD, Keisuke Sasai, MD, PhD \\ Department of Radiation Oncology, Juntendo University, Tokyo, Japan
}

\begin{abstract}
Purpose: We designed a bi-valve vaginal speculum high-dose-rate (HDR) interstitial gynecologic brachytherapy applicator. This allows for both a direct view of the cervix and image-guided brachytherapy applicator placement. The purpose of this study was to assess the validity of the new applicator.

Material and methods: The applicator was designed to have a 25-mm arc, which can be spread transversely to 35-mm wide, with 10 insertion holes; it was produced using a stereolithographic printer with biocompatible Dental SG resin. For resin radiodensity was measured in Hounsfield units (HU) using computed tomography (CT). Comparing the new applicator with a conventional intracavitary applicator, we evaluated the treated volume (including dimensions of $100 \%$ isodose volume at the central axis), $\mathrm{V}_{100} / \mathrm{D}_{90} / \mathrm{D}_{98}$ for a hypothetical cervix (a 2-cm-long and 4-cm-diameter cylinder), and dose points of organs at risk (OARs) (at 25 and $30 \mathrm{~mm}$ from the tandem). Based on dose-volume histogram (DVH) analysis of the cervix and dose points of OARs, the range of tolerance for the percent dose difference in the prescription dose was set at $5 \%$.

Results: The mean radiodensity of the Dental SG resin, which was magnetic resonance imaging compatible, was $118 \mathrm{HU}$. Dimensions of the $100 \%$ isodose volume measured at the central axis were $4.4 \times 6.6 \times 7.4 \mathrm{~cm}$ for the new applicator and $4.3 \times 6.0 \times 7.7 \mathrm{~cm}$ for the intracavitary applicator. The $100 \%$ prescription dose volumes were $110 \mathrm{cc}$ and $113 \mathrm{cc}$ for the new and conventional applicator, respectively. The percent difference in the hypothetical cervix $\mathrm{V}_{100}, \mathrm{D}_{90}$, and $\mathrm{D}_{98}$ between the new and intracavitary applicator were within $5 \%$. The percent differences in dose points of OARs at 25 and $30 \mathrm{~mm}$ between the new and conventional applicators were within $5 \%$.

Conclusions: Our speculum applicator can reproduce a conventional pear-shaped dose distribution. Our current clinical practice will use this applicator, which can improve the patient's treatment results.

J Contemp Brachytherapy 2020; 12, 1: 27-34 DOI: https://doi.org/10.5114/jcb.2020.92406
\end{abstract}

Key words: gynecologic applicator, interstitial brachytherapy applicator, 3D printing.

\section{Purpose}

High-dose-rate (HDR) interstitial brachytherapy (ISBT) has been used in definitive gynecologic radiotherapy in patients with advanced cervical carcinoma [1] Significant improvements in survival have been reported with the addition of image guidance [2]. Excellent clinical outcomes have been achieved with HDR ISBT using computed tomography (CT)-guided catheter placement for gynecologic cancer patients [3], and we routinely perform this procedure at our institution.

In ISBT with CT-guided catheter placement, we use an iterative approach of catheter implantation and adjustment with serial image acquisition, which is similar to the real-time CT-guided approach of Lee et al. [3]. After CT images are acquired to evaluate the initial catheter position, catheter adjustment and additional catheter implan- tation are performed with serial image acquisition to ensure that the tumor is fully encompassed by the catheters. To avoid generating a cold spot, based on the position of initial catheters including a vaginal fornix applicator (e.g., the ovoid applicator), both insertion points and directions of additional needles at target sites can be determined.

To identify the appropriate location of additional needles, both insertion points and directions of additional needles are determined while confirming the positional relationship between catheters by direct visualization. However, in this situation, the conventional vaginal fornix applicator does not have visible insertion points due to its placement within the vagina. This lack of visibility of the cervix due to the presence of the vaginal applicator makes it difficult to identify the insertion point by direct visualization. 
To address this problem, we designed a new bi-valve vaginal speculum applicator that allows for both a direct view of the cervix and CT-guided catheter placement. Our ISBT applicator also includes an intracavitary applicator. This should improve the intracavitary dose distribution through use of additional interstitial needle applicators under CT guidance, while expanding the entire vaginal canal and visually confirming the positional relationship between the needle tip and the tumor location or enabling a clear positional relationship between catheters.

To assess the validity of the bi-valve vaginal speculum HDR brachytherapy applicator in our current clinical practice, we explored whether conventional pear-shaped isodose distribution is achieved using the novel speculum brachytherapy applicator and tandem and ovoid conventional applicators.

\section{Material and methods}

\section{Applicator design}

A novel brachytherapy applicator was designed to have a shape similar to a bi-valve vaginal speculum, which allows the vaginal cavity to be opened and allows for a clear view of the cervix. The bi-valve vaginal speculum applicator was also designed to have interstitial multi-catheter holes, similar to the MUPIT or Syed-Neblett applicator $[4,5]$, for interstitial brachytherapy. Based on the MUPIT or Syed-Neblett cylinder applicator [4,5], the bi-valve design of the new applicator has a partially cylindrical shape. The interstitial multi-catheter holes (small circular arc grooves for insertion) in the speculum applicator are arranged on the outer surface of the partial cylindrical shape at equal intervals so that the interval becomes approximately $5 \mathrm{~mm}$ (5 holes in each valve, 10 holes in total). The diameter of the insertion holes was designed to accommodate standard after-loading catheters (o.d. 5 or 6 French). The bi-valve vaginal speculum applicator can be spread transversely during use. In a clinical setting, the inside of a vaginal speculum applicator is generally filled with water jelly using gauze. Therefore, the effect of air inside the vaginal speculum applicator is negligible.

A vaginal speculum applicator with needle insertion holes can be generated using the computer-aided design (CAD) software Fusion 360 (Autodesk, Inc., San Francisco, CA, USA), as shown in Figure 1A-C. The bi-valve vaginal speculum applicator was produced using a laptop and stereolithographic printer (Form 2; Formlabs, Inc.), coupled with synergistic biocompatible resin (Dental SG; Formlabs, Inc.), which is certified to comply with the international standards for class I biocompatibility, International Organization for Standardization (ISO) standard 10993-1. This 3D printing technology has become popular in implant dentistry for constructing surgical guides [6,7]. As shown in Figure 1D, the 3D prints had a thickness of $0.05 \mathrm{~mm}$ per layer. After printing, the resins were washed using anhydrous ethanol for $30 \mathrm{~min}$. To maximize printed material strength, the materials were exposed to ultraviolet radiation for $30 \mathrm{~min}$ at a temperature of $60^{\circ} \mathrm{C}$ using Form Cure (Formlabs, Inc). In this study, to fit the aver- age Japanese patient's vaginal size, the bi-valve vaginal speculum applicator was designed to have a $25-\mathrm{mm}$ arc, which can be spread transversely to $35 \mathrm{~mm}$, with 10 insertion holes of 3-mm in diameter (Figure 1), although a custom-sized, optimal bi-valve vaginal speculum applicator size can be designed and generated by modifying the CAD data to fit the patient's vaginal size. The actual distance between the centers of the multi-catheter holes of the new applicator was approximately $5 \mathrm{~mm}(4.6 \mathrm{~mm})$, similar to the distance between the dwell positions in the ovoid of the conventional intracavitary applicator. Figure 2 shows the closed and opened states of the speculum applicator. Each partially cylindrically shaped blade has a hinge structure, similar to that of the vaginal speculum. The speculum applicator is introduced into the vagina with the two blades closed, which facilitates its insertion; the two blades of the applicator are then spread transversely and maintained in an open position by fixation with a thread.

\section{Evaluation of the radiation attenuation properties of biocompatible resin for use in a 3D-printed brachytherapy applicator}

For evaluation of the synergistic biocompatible resin (Dental SG; Formlabs, Inc.), CT was performed (1-mm slice thickness; Aquilion LB; Canon Medical Systems, Tochigi, Japan). The radiodensity in Hounsfield units (HU) was measured in an $8 \times 8 \times 8$-voxel area of a Dental SG resin testing apparatus.

\section{High-dose-rate brachytherapy planning}

We used a hypothetical uterine cervix for HDR brachytherapy planning. Based on comparison of the new and conventional applicators, we produced each dose distribution using the new or conventional intracavitary applicator. As the conventional intracavitary applicator, we used the ovoid of the unshielded specific Fletcher Williamson HDR applicator (Elekta Instrument $\mathrm{AB}$, Stockholm, Sweden), the most commonly used applicator in Japan.

CT scans of the applicator were acquired with 1-mm slice spacing. Three-dimensional planning was performed on the Elekta Nucletron Oncentra Planning System (Version 4.3; Elekta Instrument AB, Stockholm, Sweden). HDR brachytherapy planning was generated using an ${ }^{192}$ Ir source. To simplify the comparison, a straight tandem with 5-cm active length was used for planning each applicator (Figure 3). For DVH analysis, the hypothetical cervix was defined as a 2-cm-long, 4-cm-diameter cylinder centered along the tandem.

During vaginal speculum applicator planning for the hypothetical target, catheter reconstruction was simulated using catheter holes without insertion of interstitial needles. Eleven catheters were used, including 1 inserted centrally along the tandem. The vaginal speculum applicator was spread transversely so that the width at the outermost needle tips was $35 \mathrm{~mm}$. Intracavitary applicator planning was generated using mini-size ovoid applicators (Ovoid Pair, Mini; Elekta Instrument AB, Stockholm, 

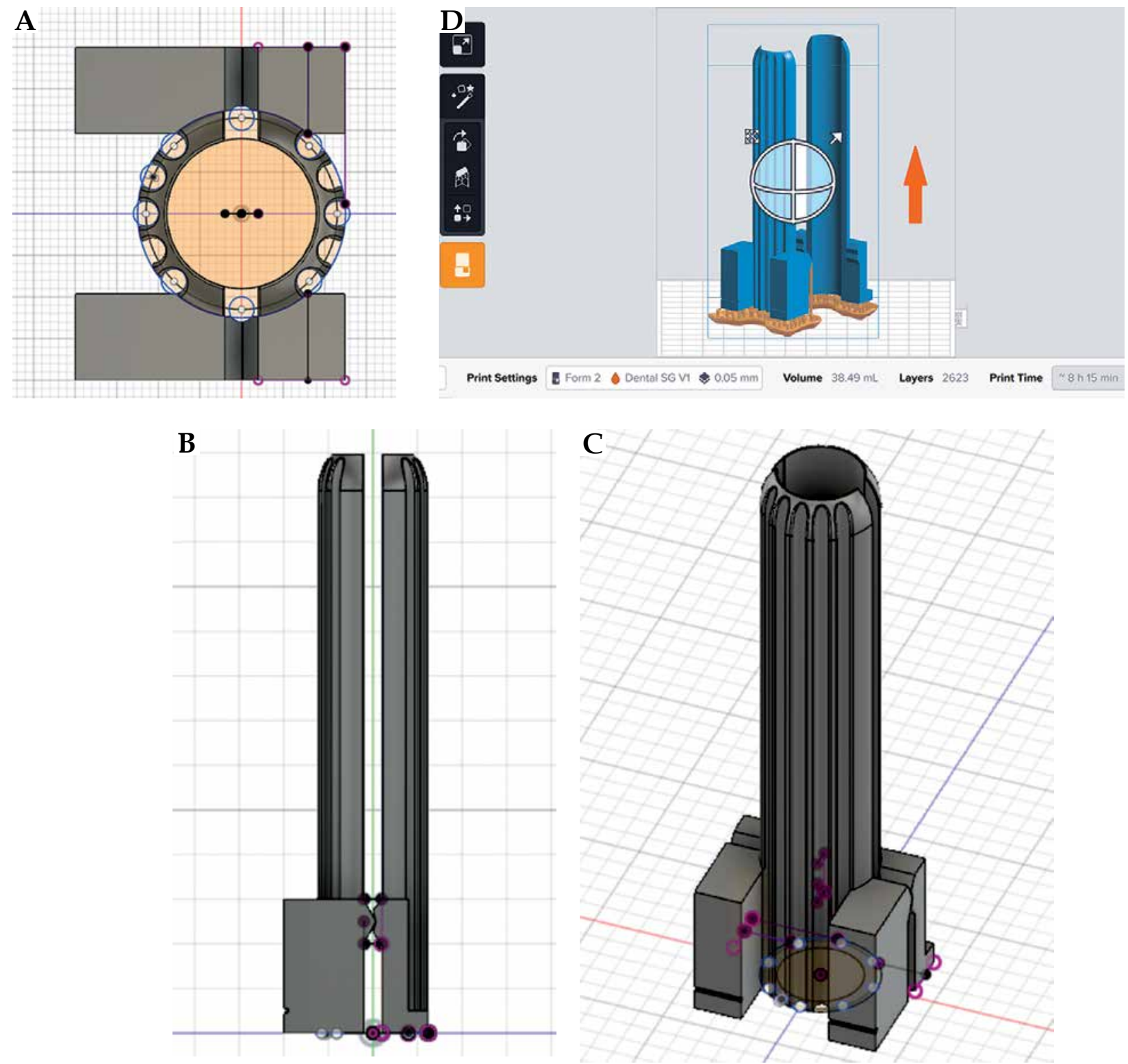

Fig. 1. Projection views and bird's eye view of speculum applicator generated using computer-aided design (CAD) software (A-C) and the print settings of the speculum applicator (D). (A) Top, (B) side, and (C) bird's eye views of the applicator are shown. The bi-valve vaginal speculum applicator is designed to have a 25-mm arc with 10 insertion holes of $3 \mathrm{~mm}$ in diameter and with a fixed groove to hook a thread. Each speculum blade has a hinge structure similar to that of the vaginal speculum. During the 3D printing setup of the speculum applicator (D), the prints were set to have a thickness of $0.05 \mathrm{~mm}$ per layer (in the direction indicated by the orange arrow) and the print supports were generated

Sweden). Each ovoid applicator was spread transversely to $35 \mathrm{~mm}$. Ovoid applicators with 2 -cm active length were used.

In each plan, 11 dwell positions were activated every $5 \mathrm{~mm}$ along each tandem. In the peripheral insertion holes of the speculum applicator plan, the first dwell position at the tip of each hole was activated ( $10 \mathrm{dwell}$ positions in total). In each ovoid of the intracavitary plan, $5 \mathrm{dwell}$ positions were activated at intervals of $5 \mathrm{~mm}$ along each ovoid (10 dwell positions in total). Each tandem had the same number of dwell positions, and the total number of dwell positions in each plan was the same. The dwell times of all sources were evenly weighted to produce the pear-shaped isodose distribution in each plan. The prescribed dose was administered to Point A (pA). Figure 3 illustrates both plans. No manual adjustment of dwell time or graphical optimization was performed.

\section{Verification of bi-valve vaginal speculum brachytherapy applicator accuracy}

It is important to verify whether a new applicator can provide a pear-shaped dose distribution equivalent to that with a conventional tandem/ovoid applicator. To compare the new and conventional applicator planning, we evaluated treated volume (including the dimensions 

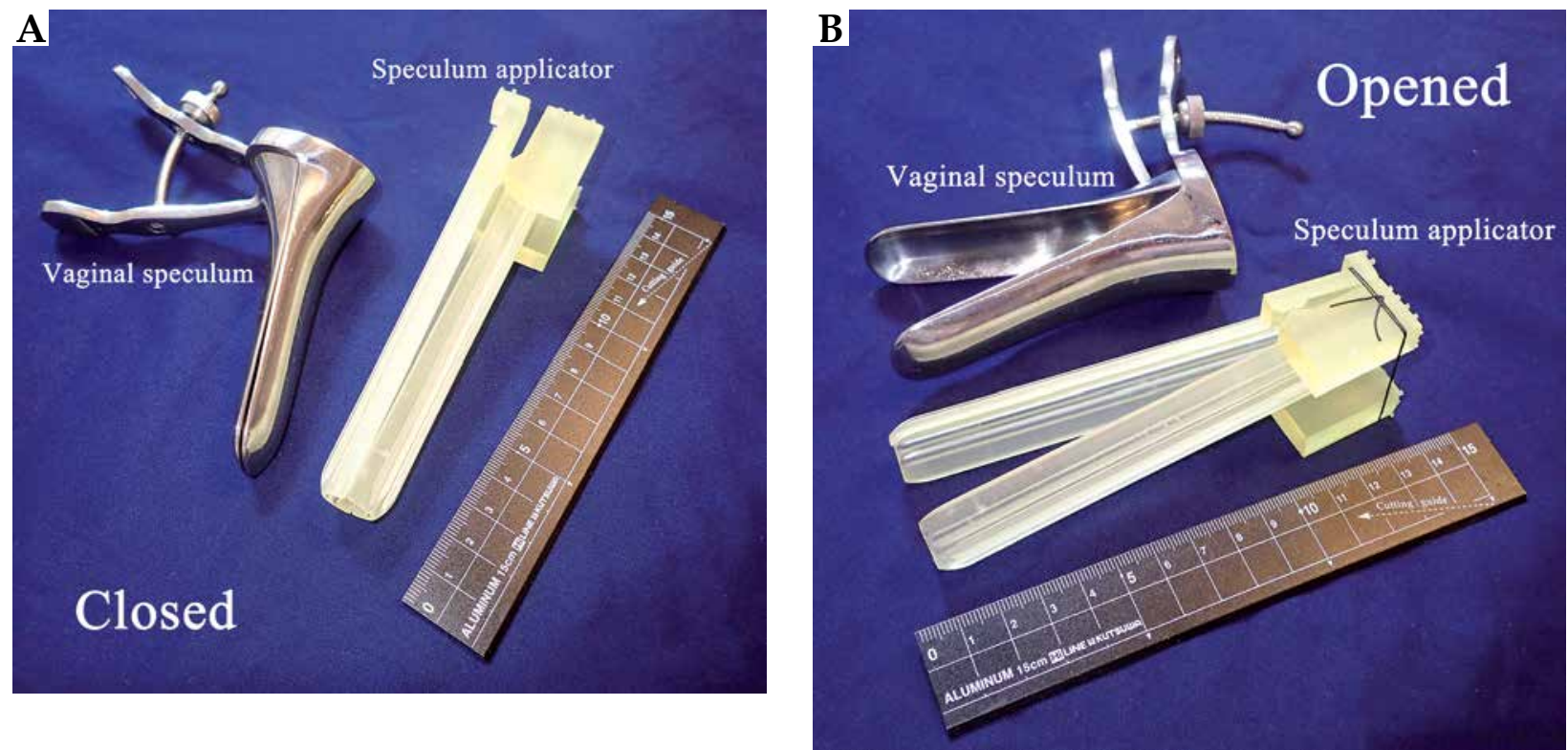

Fig. 2. The 3D-printed speculum applicator after polymerization. Each partially cylindrically shaped blade has a hinge structure, similar to that of the vaginal speculum. The applicator is shown with both blades closed (A), which facilitates its insertion, and opened (B), to allow a direct view of the cervix to be obtained. During implantation (B), the blades of the 3D-printed speculum applicator can be spread transversely to $35-\mathrm{mm}$ wide, and then fixed via a black thread fed through the groove to maintain the applicator in an open position

of the $100 \%$ isodose volume), the dose-volume histogram (DVH) for a hypothetical cervix, and the dose points of organs at risk (OARs), such as the bladder and rectum. The dimensions of the $100 \%$ isodose volume were measured at the central axis.

In DVH analysis, the following metrics were used: percentage of the hypothetical target covered by $100 \%$ of the prescription dose $\left(\mathrm{V}_{100}\right)$ and minimum dose received by $90 \%$ and by $98 \%$ of the hypothetical target, expressed as a percentage of the prescription dose $\left(D_{90}\right.$ and $D_{98}$, respectively).

To evaluate and compare doses to OARs, dose points of OARs in each plan were plotted at 25 and $30 \mathrm{~mm}$ from the tandem in the inferior plane of the hypothetical cervix.

Based on DVH analysis of the cervix and dose points of OARs, the range of tolerance in the percent dose difference in the prescription dose was set at within $5 \%$.

\section{Results}

\section{Radiation attenuation properties of biocompatible resin}

No CT artifacts were visible inside the speculum applicator. The mean radiodensity of the Dental SG resin was $118 \mathrm{HU}$ (standard deviation: $10 \mathrm{HU}$ ). This biocompatible Dental SG resin was therefore confirmed to be magnetic resonance imaging-compatible.

\section{Comparison of treatment volume, $\mathrm{DVH}$, and dose points of $O A R s$}

Dimensions of the $100 \%$ isodose volume were measured at the central axis for each plan. The dimensions were $4.4 \times 6.6 \times 7.4 \mathrm{~cm}$ for the new applicator and
$4.3 \times 6.0 \times 7.7 \mathrm{~cm}$ for the intracavitary applicator. The $100 \%$ prescription dose volumes were $110 \mathrm{cc}$ and $113 \mathrm{cc}$ for the new and conventional applicator, respectively.

The treatment volumes receiving $100 \%, 110 \%, 120 \%$, $130 \%, 140 \%, 150 \%, 160 \%, 170 \%, 180 \%, 190 \%$, and $200 \%$ of the prescription dose for the new and conventional applicator are plotted in Figure 4.

Percentages of the hypothetical cervix receiving a higher dose in each applicator plan are shown in Figure 5 . The percent differences in $V_{100}, D_{90}$, and $D_{98}$ between the new and intracavitary applicator were within $5 \%$. Figure 6 shows dose points of OARs plotted at 25 and $30 \mathrm{~mm}$ for the new and intracavitary applicators. Percent dose differences between the new and conventional applicator at each point were within $5 \%$.

\section{Discussion}

Better target coverage in ISBT depends on the accuracy of catheter positioning. Image guidance is required to accurately implant catheters. Direct visibility of the gross tumor is also critical when radiation oncologists perform catheter implantation. Image-guided placement with the addition of a real-time direct view of the cervix using our speculum applicator can improve the quality of catheter positioning and, by extension, clinical outcomes. No ISBT applicator allowing for a direct view is commercially available at present, and existing applicators do not provide a direct view and are inconvenient for radiation oncologists. Our bi-valve vaginal speculum applicator resolves this problem. Thus, we believe that our novel speculum applicator will have a considerable impact on patient treatment outcomes when an intracavitary applicator is insufficient to cover the tumor, such that an 

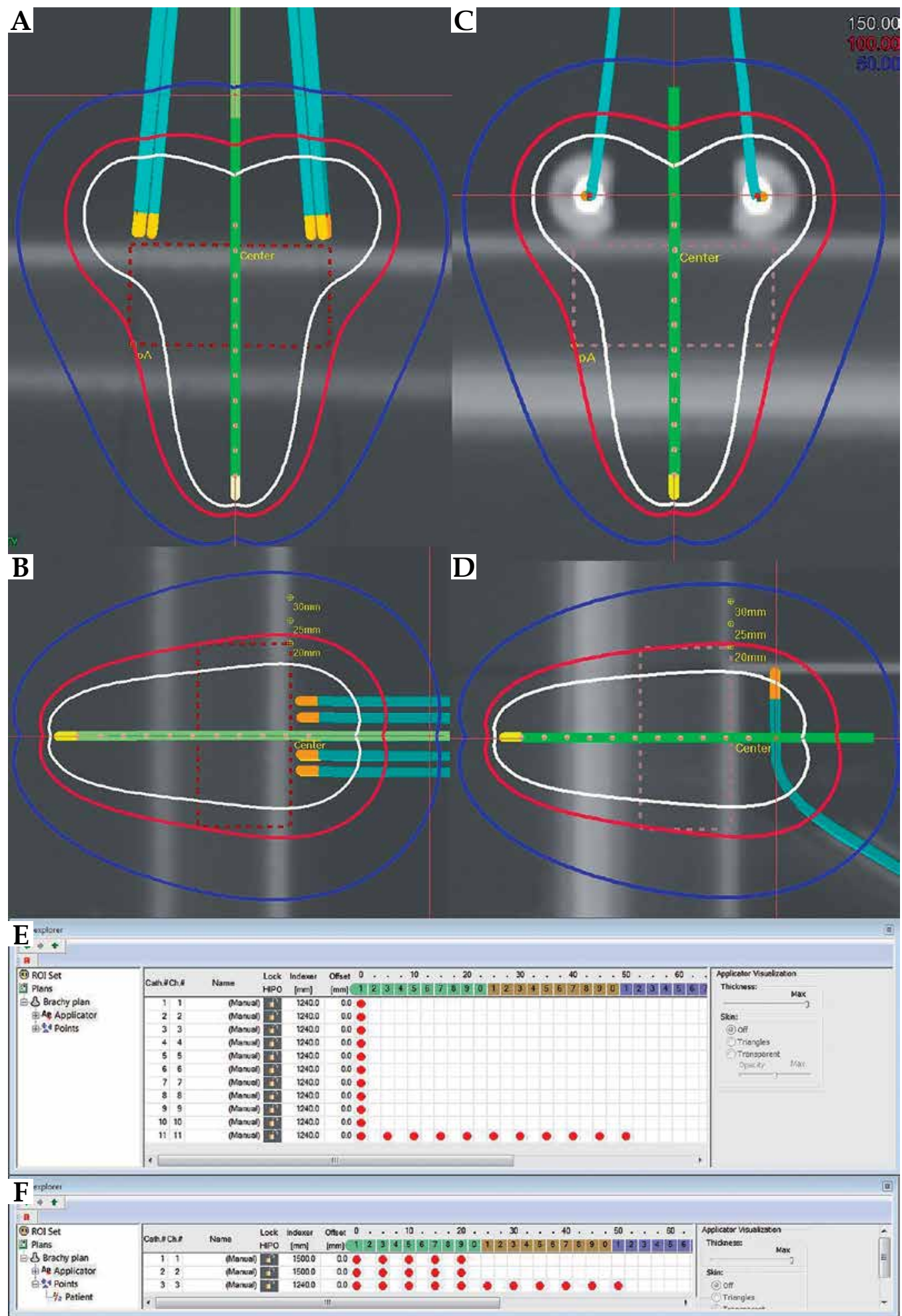

Fig. 3. Pear-shaped isodose distribution in the speculum applicator and conventional applicator. Each applicator can be spread transversely to $35 \mathrm{~mm}$ wide, and the $100 \%$ dose prescription is administered to Point A (pA). (A) Coronal and (B) sagittal views of the speculum applicator plan and (C) coronal and (D) sagittal views of the conventional applicator plan. During speculum applicator planning (E), the first dwell position at the tip (10 dwell positions in total), and $11 \mathrm{dwell}$ positions (every $5 \mathrm{~mm}$ ) along the tandem, are activated. In conventional applicator planning (F), 5 dwell positions at intervals of $5 \mathrm{~mm}$ along each ovoid (10 dwell positions in total) and 11 dwell positions (every $5 \mathrm{~mm}$ ) along the tandem are activated. The dose distributions are shown in blue lines $(50 \%)$, red lines $(100 \%)$, and white lines $(150 \%)$. The hypothetical cervix (dotted red or pink rectangular ranges) receives a dose of approximately $100 \%$. In each applicator plan, the reconstructed catheters are shown. The speculum applicator can reproduce conventional pear-shaped isodose distributions, similar to the conventional applicator 


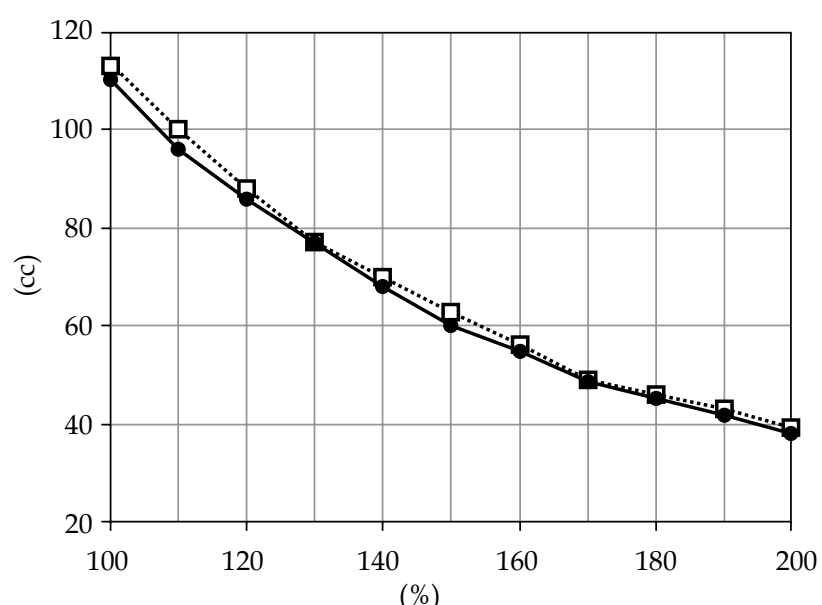

Fig. 4. Treatment volumes receiving 100\%, $110 \%, 120 \%$, $130 \%, 140 \%, 150 \%, 160 \%, 170 \%, 180 \%, 190 \%$, and $200 \%$ of the prescription dose plotted for the new applicator (solid line) and for the conventional applicator (dotted line). The treatment volumes appear to be overlapping, including the higher dose volumes

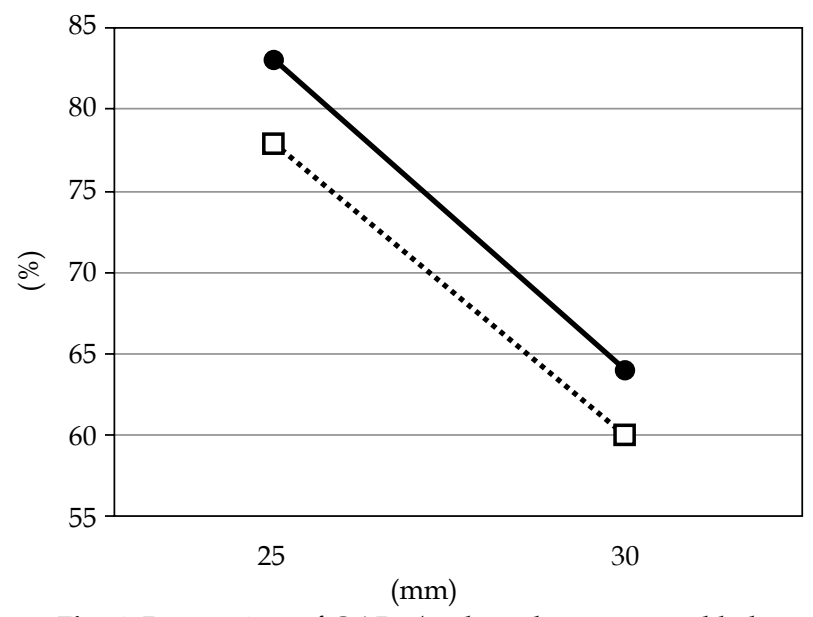

Fig. 6. Dose points of OARs (such as the rectum or bladder) plotted at 25 and $30 \mathrm{~mm}$. Percent dose differences between the new (solid line) and conventional (dotted line) applicator at each point were within $5 \%$

additional interstitial technique could improve treatment results. However, we acknowledge the greater degree of trauma associated with interstitial techniques, and the increased size of high-dose areas, as seen when commercially available ISBT applicators are used.

In HDR ISBT using our novel speculum applicator, which provides a view of the cervix, we can implant interstitial brachytherapy needles at the intended positions. We can then leave the speculum applicator in place and irradiate a target without the deformation caused by the exchange of a vaginal speculum for a vaginal fornix applicator. In HDR ISBT, a vaginal fornix applicator is placed after needle implantation, or needles are inserted after placement of a vaginal fornix applicator. The former can generate deformation caused by the vaginal fornix applicator, and the latter can result in poor visibility of the cervix due to the vaginal applicator. Deformation of

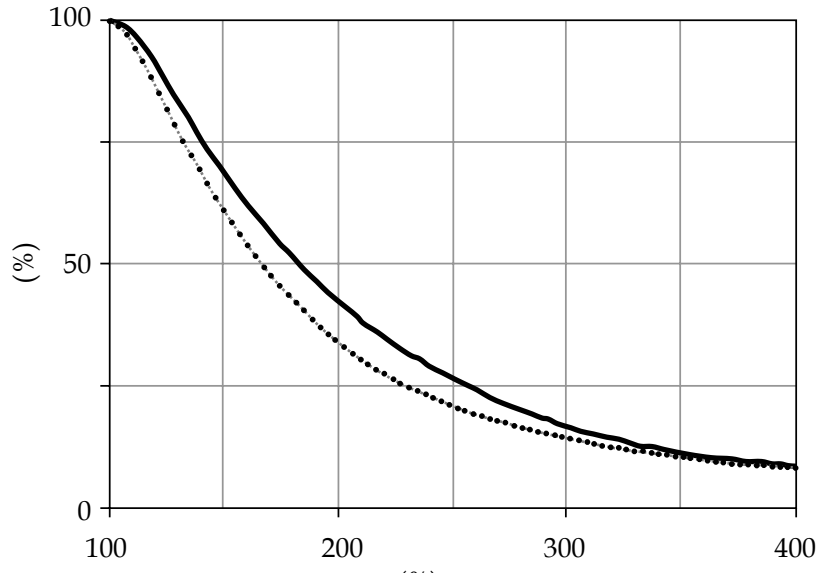

$(\%)$

Fig. 5. Dose volume histogram of the hypothetical cervix for the new applicator (solid line) and for the conventional applicator (dotted line). The percent differences in the hypothetical cervix $\mathrm{V}_{100}, \mathrm{D}_{90}$, and $\mathrm{D}_{98}$ between the new and intracavitary applicator were within $5 \%$. The volume for the higher doses (e.g., $150 \%$ or $200 \%$ ) is slightly higher for the new applicator than for the conventional applicator.

the vaginal fornix may decrease the dose coverage of the clinical target volume. The view of the cervix ensured by a speculum can guide the brachytherapy catheters to the intended positions.

The development of a brachytherapy applicator is important for improved target coverage. To improve brachytherapy dose distributions, many investigators have proposed novel applicator designs and methods, including 3D-printed custom-made vaginal cylinders, vaginal vault brachytherapy with a CT imaging-derived applicator, an MRI-compatible intracavitary/interstitial gynecologic brachytherapy applicator, an inversely designed 3D-printed personalized template-guided interstitial vaginal applicator, and 3D-printed HDR skin brachytherapy applicators $[8,9,10,11,12]$.

In this study, the validity of the new applicator was verified by comparison of the pear-shaped isodose distribution. Our novel ISBT applicator is combined with an intracavitary applicator; hence, it should be considered an advanced intracavitary applicator. Radiation oncologists will be able to exploit the direct view capability of our applicator to improve the conventional pear-shaped dose distribution, via use of additional interstitial needle applicators, thus enhancing target coverage. If necessary, radiation oncologists will also be able to insert an ISBT applicator into the target via fixed insertion holes within the two speculum blades. To determine the validity of our combined intracavitary and ISBT applicator, we compared its dose distribution to the dose distribution typical of conventional intracavitary applicators (e.g., tandem plus ovoids). The main goal of this study was to assess whether our proposed speculum applicator could consistently generate a conventional pear-shaped dose distribution. The results showed that the difference in dosage between the vaginal speculum brachytherapy applicator and the intracavitary brachytherapy applicator was min- 
imal in a clinical setting. There was a minimal difference in the dimensions of the $100 \%$ isodose volume. Visual inspection of Figure 4 demonstrates that the treatment volumes overlapped, including the higher-dose volumes.

There is a difference of several millimeters in the dwell position between the vaginal speculum and ovoid applicators. Although the number of dwell positions in the new speculum planning was equal to that of conventional planning, the distance between the dwell position and the inferior plane of the hypothetical cervix was $6 \mathrm{~mm}$ for the new applicator and $9 \mathrm{~mm}$ for the conventional applicator. Due to this difference, visual inspection of the dose distributions showed that the new applicator had larger volumes with a higher dose (such as 150\% isodose, shown in Figure 3) around the inferior plane of the hypothetical cervix compared to the conventional applicator. Visual inspection of Figure 5 reveals that the dose volume histograms for the hypothetical cervix overlapped, although the volume of the higher dose (e.g., $150 \%$ or $200 \%$ ) using the new applicator was slightly greater than that with the conventional applicator. With regard to the central higher dose to the hypothetical cervix, we allowed for the larger volume of the new applicator (Figures 3, 5) because the potentially radioresistant tumor cells of cervical cancer are often centrally located around the cervical os.

We performed a preliminary dosimetric analysis of the speculum applicator to demonstrate a conventional pear-shaped dose distribution and support the clinical usefulness of this novel applicator.

After demonstrating that the new speculum applicator can improve patient treatment outcomes, we used it to treat cervical cancer that had spread to the lower part of the vagina. In such cases, it is difficult for a standard ovoid applicator to cover the lower lesion of the vagina, and for standard cylinder applicators (such as the MUPIT) to fit onto the vaginal fornix (because the vaginal fornix is wider than the vaginal opening). Therefore, we used our speculum applicator and introduced it into the vagina with both blades closed to facilitate insertion; this procedure was performed in a room with a dedicated CT scanner capable of scanning a patient in the lithotomy position. After introduction, the two blades of the applicator were spread transversely and maintained in an open position by fixation with a thread. The applicator was loosely fixed to the vagina. Subsequently, transrectal ultrasound (TRUS)- and CT-guided catheter placement, with visualization of the cervix, was performed using flexible needles (o.d. 5 French, ProGuide Sharp Needle; Nucletron B.V., Veenendaal, The Netherlands). As shown in Figure 7, a non-central intrauterine plastic catheter, and an additional plastic needle emanating from inside the two speculum blades, were implanted under the direct view. Six plastic catheters were easily inserted, via interstitial holes, into the cervix. After all catheters had been implanted, the inside of the vaginal speculum applicator was filled with water jelly using gauze. The applicator was sutured to the perineal skin with a thread to avoid displacement; planning CT was then performed. The HDR brachytherapy plan was generated using the Nucletron Oncentra treatment planning system, with the inverse

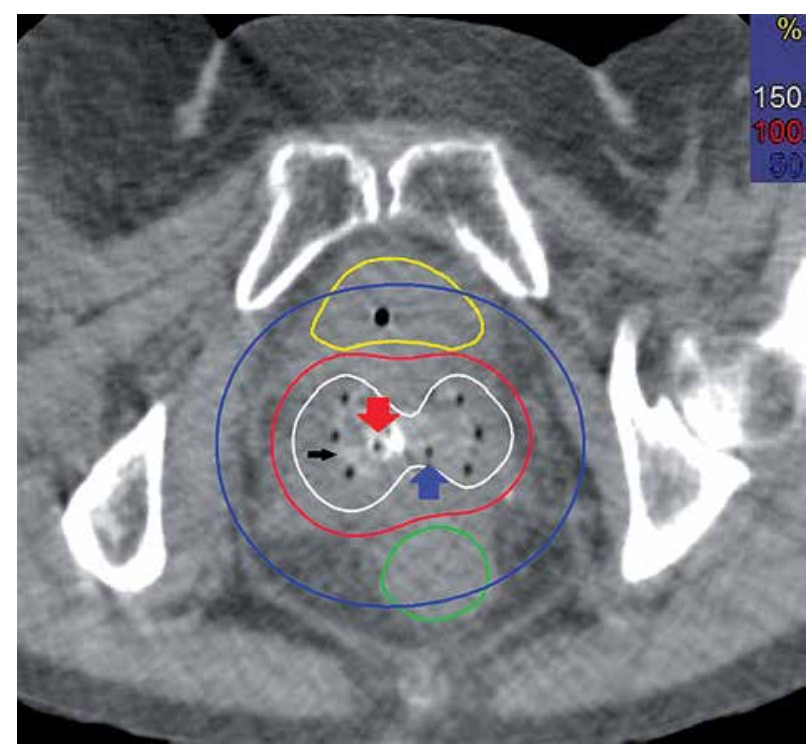

Fig. 7. Axial computed tomography (CT) image of the bladder (yellow line), rectum (green line), and cervix (between bladder and rectum) receiving 50\% (blue lines), $100 \%$ (red lines), and 150\% (white lines) of the prescription dose in a patient with cervical cancer. The Dental SG resin applicator (black arrow) can easily be visualized (mean radiodensity: $118 \mathrm{HU}$ ) in the vaginal fornix. An intrauterine plastic catheter (red arrow) is introduced into the non-central intrauterine cervix, and an interstitial flexible catheter (blue arrow) is implanted into the cervix from inside the speculum applicator. Reconstruction of the plastic catheters was performed using the inner air defects of the catheters (o.d. 5 French). The high-dose-rate brachytherapy plan was generated in the Nucletron Oncentra treatment planning system using the inverse planning simulated annealing (IPSA) optimization algorithm

planning simulated annealing (IPSA) optimization algorithm applied (and without any steps related specifically to the use of the speculum applicator; Figure 7). The patient was treated in the lithotomy position on the CT table, and remained still after implantation; the implant was then removed.

A Dental SG resin speculum applicator exhibits less reflection than a stainless steel speculum. To address this issue, a light is needed to view the cervix. Our bi-valve vaginal speculum applicator can be used in advanced cervical cancer that has spread to the lower part of the vagina, whereas the standard ovoid applicator does not always cover the lower lesion of the vagina. Future studies should evaluate the usefulness of our applicator using different hypothetical target models such as cervical cancer that has spread to the lower part of the vagina. In future studies, we will compare our applicator with other applicators including interstitial components.

\section{Conclusions}

We designed a novel vaginal speculum applicator with needle insertion holes for combined intracavitary and interstitial GYN HDR brachytherapy. Our speculum 
applicator, which allows for combined observation of the cervix through direct and imaging views for accurate catheter positioning and is produced using a 3D printer with biocompatible resin, can reproduce a conventional pear-shaped dose distribution. Our current clinical practice will use this applicator, which can improve patient treatment results.

\section{Acknowledgements}

This work was supported in part by JSPS KAKENHI Grant Number 16K19857 and 18K15562.

\section{Disclosure}

The authors report no conflict of interest.

\section{References}

1. Pötter R, Georg P, Dimopoulos JC et al. Clinical outcome of protocol based image (MRI) guided adaptive brachytherapy combined with 3D conformal radiotherapy with or without chemotherapy in patients with locally advanced cervical cancer. Radiother Oncol 2011; 100: 116-123.

2. Viswanathan AN, Erickson BA. Seeing is saving: the benefit of 3D imaging in gynecologic brachytherapy. Gynecol Oncol 2015; 138: 207-215.

3. Lee LJ, Damato AL, Viswanathan AN. Clinical outcomes of high-dose-rate interstitial gynecologic brachytherapy using real-time CT guidance. Brachytherapy 2013; 12: 303-310.

4. Rodríguez Villalba S, Richart Sancho J, Otal Palacín A, et al. Development and clinical implementation of a new template for MRI-based intracavitary/interstitial gynecologic brachytherapy for locally advanced cervical cancer: from CTbased MUPIT to the MRI compatible Template Benidorm. Ten years of experience. J Contemp Brachytherapy 2016; 8: 404-414.

5. Stock RG, Chan $K$, Terk $M$ et al. A new technique for performing Syed-Neblett template interstitial implants for gynecologic malignancies using transrectal-ultrasound guidance. Int J Radiat Oncol Biol Phys 1997; 37: 819-825.

6. Katkar RA, Taft RM, Grant GT. 3D Volume Rendering and 3D Printing (Additive Manufacturing). Dent Clin North Am 2018; 62: 393-402.

7. Goodacre BJ, Swamidass RS, Lozada J et al. A 3D-printed guide for lateral approach sinus grafting: A dental technique. J Prosthet Dent 2018; 119: 897-901.

8. Sethi R, Cunha A, Mellis K et al. Clinical applications of custom-made vaginal cylinders constructed using three-dimensional printing technology. J Contemp Brachytherapy 2016; 8: 208-214.

9. Wiebe E, Easton $\mathrm{H}$, Thomas $\mathrm{G}$ et al. Customized vaginal vault brachytherapy with computed tomography imaging-derived applicator prototyping. Brachytherapy 2015; 14: 380-384.

10. Rodriguez Villalba S, Richart Sancho J, Otal Palacin A et al. A new template for MRI-based intracavitary/interstitial gynecologic brachytherapy: design and clinical implementation. J Contemp Brachytherapy 2015; 7: 265-272.

11. Sekii S, Tsujino K, Kosaka $\mathrm{K}$ et al. Inversely designed, 3D-printed personalized template-guided interstitial brachytherapy for vaginal tumors. J Contemp Brachytherapy 2018; 10: 470-477.

12. Jones EL, Tonino Baldion A, Thomas $C$ et al. Introduction of novel 3D-printed superficial applicators for high-dose-rate skin brachytherapy. Brachytherapy 2017; 16: 409-414. 